departments $(\mathrm{n}=17)$. Most often, a nurse undertook daily (71\%) or weekly $(14 \%)$ systematic verification of endotracheal intubation equipments in 87 emergency departments (94.6\%). The necessary drugs for the performance of endotracheal intubation and that were present in emergency rooms were: (1) midazolam, 100\%; (2) diazepam, 98.9\%; (3) thiopental, 83.3; (4) propofol, 83.3\%; (5) fentanyl, 81.1\%; (6) succinylcholine, 77.8\%; (7) etomidate, 77.8\%; (8) ketamine, $57.8 \%$; vecuronium, $55.6 \%$; (9) rocuronium, $31.1 \%$; and (10) other non-depolarizing agents, $26.7 \%$. In case of difficult airway management, the emergency practitioners found these other supplies available in emergency rooms: (1) kit for cricothyroidotomy, 69.7\%; (2) catheter for percutaneous transtracheal ventilation_; (3) kit for retrograde intubation, 21.3\%; (4) intubating laryngeal mask airway, $21.3 \%$; (5) combitube, $14.6 \%$; (6) fibroscope, $14.6 \%$; and (7) fast-track, $12.4 \%$.

Conclusion: Anaesthetic agents are present in most of emergency departments. These emergency departments are less equipped with equipment and supplies for difficult airway techniques. The presence of airway management protocols or guidelines are rare. However, in France, it is necessary to improve the endotracheal intubation training of emergency physicians. In this training, the practitioners also must learn rapid sequence intubation (RSI), which is a standard emergency department procedure, and new airway devices such as the intubating laryngeal mask airway and a Bullard laryngoscope bade.

Key words: airway management; anesthetic agents; cricothyrotomy; drugs; endotracheal intubation; equipment; emergency departments; rapid sequence intubation; supplies; ventilation

Prehosp Disast Med 2001;16(2):s70.

\section{Consumption and Cost of Prescribed Medicines in Eight Emergency Departments in France and in Switzerland \\ F. Staikowsky; ${ }^{1}$ A. Roumy; ${ }^{1}$ I. Billottet; , F. Carpentier; ${ }^{2}$ D. Baron;, G. Bleichner; ${ }^{4}$ P. Kiege, 5 P.F. Unger; and the Société Francophone de Médecine d'Urgence Board \\ Emergency Departments: 1. Caen; 2. Grenoble; 3.} Argenteuil; 4. Aix en Provence; 5. Genève; 6. Marseille, FRANCE

Introduction: Monitoring of consumption and costs of prescribed medicines in emergency department (budgetary monitoring) are often unknown by the emergency teams. This work analyzes these data in different emergency departments.

Methods: For each emergency department, the annual (1999) medicine expenses have been classified according to two modes: (1) quantitative by decreasing order the 20 first per os administered products on the one hand, and the first 20 intravenously or intramuscularly administered products; (2) by cost by decreasing the orders for the 20 most expensive products. For the global quantitative analysis, the products have been classified according to their frequency of quotation in each 20 first administered products list. Results: Eight emergency departments were enrolled in the study (seven in France and one in Switzerland). Characteristics of these emergency departments were: five teaching departments, two medical and six trauma-medical emergency centers, with a short-term hospitalisation unit in five emergency departments. For this study, the average number of annual admissions was $43,150 \pm 19,360 /$ year (400,000 annual admissions entirely). Among the 20 first per os administered products, in all emergency departments, paracetamol (acetaminophen) was the most frequently delivered product (more than 190,000 units distributed /year), and associated paracetamol and propoxyphene constituted $37 \%$ of the shares. The other 20 first per os administered products were, in descending order: phloroglucinol, aspirin, alprazolam, amoxicillin, amoxicillin-clavulanate, prednisone, omeprazole, and activated charcoal. Among the 20 first intravenously or intramuscularly administered products, the proparacetamol was the most prescribed (36,000 units of 1 and $2 \mathrm{~g}$ by year); the other most current parenteral injectable molecules in descending order were: lidocaïne, amoxicillin-clavulanate, phloroglucinol, ketoprofene, methylprednisolone, morphine, furosemide, omeprazole, metoclopramide, epinephrine, trinitrine, unfractionated heparin, and low molecular weight heparin. Among the most expensive molecules, some did not belong to quantitatively prescribed products: antiretroviral drugs, sandostatine, some antidotes (flumazenil, $\mathrm{N}$-acetyl cysteine), dobutamine, rt-PA; others already had been noted and included: amoxicillin-clavulanate, proparacetamol, omeprazole, activated charcoal, phloroglucinol, lidocaïne, and ketoprofene.

Conclusion: This multicenter evaluation is an interesting economic approach among French-speaking emergency departments. In each emergency department, this approach provides the data to create a budget monitoring of the consumption and costs of prescribed medications.

Key words: consumption; costs; drugs; emergency departments, France

Prebosp Disast Med 2001;16(2):s70.

\section{Abuse of Poppers: Four Cases of Methemoglobinemia Observed in an Emergency Room \\ F. Staïkowsky; ${ }^{1}$ C. Zanker, ${ }^{2}$ F. Pevirieri, ${ }^{2}$ C. Potier, $;$ A. Lepelletier; ${ }^{1}$ I. Dandine ${ }^{1}$ \\ Emergency Departments in 1. Caen; and 2. Paris, FRANCE}

Introduction: Methemoglobinemia is an exceptional complaint in emergency rooms. Its origin is especially toxic. Butyl and propyl nitrites "poppers" are being used increasingly as aphrodisiacs. We describe four cases of methemoglobinemia following ingestion or inhalation of nitrite poppers.

Case 1: A 36 year-old-man was admitted for cyanosis, confusion, agitation, and loss of consciousness following the ingestion of poppers. The blood pressure was 130/79 $\mathrm{mmHg}$, the pulse rate was 103 beats/min., and pulse oximeter $\left(\mathrm{SpO}_{2}\right)$ read $90 \%$ (oxygen administreation at 6 $\mathrm{V} / \mathrm{min})$. The patient presented with coma, agitation, dark 
colour of the skin, and chocolate colour of the blood. The lungs were clear. Methemoglobinemia saturation of the blood was $67 \%$. The laboratory tests included lactic acidosis $\left(\mathrm{pH}=7.32, \mathrm{HCO}_{3}^{-}=18.5\right.$ mmol. $.^{-1}, \mathrm{PaCO}_{2}=37$ $\mathrm{mmHg}$, lactate $=5.39 \mathrm{mmol} . \mathrm{l}^{-1}$ ) and $\mathrm{PaO}_{2}=340 \mathrm{mmHg}$ (oxygen $6 \mathrm{l} / \mathrm{min}$ ). After $100 \mathrm{mg}$ of intravenous methylene blue, the cyanosis disappeared and the level of consciousness became normal.

Case 2: A 31 year-old-man was admitted for dizziness and loss of consciousness following sniffs of poppers and space. The anomalies were a grey complexion, low blood pressure $(90 / 60 \mathrm{mmHg})$, pulse rate $=95$ beats $/ \mathrm{min}$., and $\mathrm{SpO}_{2}=$ $92 \%$. Otherwise, clinical findings were unremarkable. The arterial blood gases were: $\mathrm{PaO}_{2}=70.5 \mathrm{mmHg}, \mathrm{SaO}_{2} 92 \%$, and the methemoglobinemia saturation $=27 \%$. After 65 $\mathrm{mg}$ of intravenous methylene blue, the evolution was rapidly favorable.

Case 3: A 49 year-old-man was admitted for loss of consciousness after 10-12 inhalations of poppers during 30 $\min$. There was diffuse cyanosis without cardiac and lung anomalies, and $\mathrm{SpO}_{2}=89 \%$. The laboratory tests showed a $\mathrm{PaO}_{2}=77 \mathrm{mmHg}, \mathrm{SaO}_{2}=73 \%$, methemoglobinemia $=$ $22 \%$ with a normal electrocardiogram and chest $\mathrm{X}$-ray. At the end of methylene blue injection (100 $\mathrm{mg}$ intravenous), the cyanosis had disappeared and $\mathrm{SaO}_{2}$ was $96 \%$.

Case 4: A 39 year-old-man was admitted for headache, fatigue, pallor, cyanosis of lips and extremities, and low blood pressure. The examination noted a pallor, grey complexion, blood pressure $=98 / 49 \mathrm{mmHg}$, pulse rate $=85$ beats $/ \mathrm{min}$., $\mathrm{SpO}_{2}=92 \%$ without cardiac or lung anomalies. The electrocardiogram showed a raised ST segments in V2-V4. Arterial blood gases showed $\mathrm{PaO}_{2}=68 \mathrm{mmHg}$ and $\mathrm{SaO}_{2}=92 \%$ with normal chest X-ray. Methemoglobinemia was not administered because the diagnosis was not evoked. Later, the patient confessed the inhalation of poppers that provoked palpitations and a loss of consciousness before his arrival to emergency rooms.

Discussion: The poppers contain nitrate of propyl or butyl, causing vasodilation. Ingestion or inhalation of these products can produce a methemoglobinemia that may be fatal. Methemoglobinemia occurs when the concentration of methemoglobin in the erythrocytes is greater than $1 \%$. Because of the potential toxicity of the methylene blue, the patients, symptomatic or not, having a methemoglobinemia saturation of $>30 \%$ and symptomatic patients (other that cyanosis) with a rate $<30 \%$ should be treated with methylene blue.

Key words: aphrodisiacs; clinical manifestations; methemoglobinemia; methylene blue; nitrites; poppers

Prehosp Disast Med 2001;16(2):s71.

\section{Examining Networks of Care Proposed by Emergency Departments in France to Patients that have Attempted Suicide \\ F. Staikowsky ${ }^{1}$ and the Study Group on Suicide Attempts in Emergency Wards (GETSSU) \\ 1. Emergency Department, Caen, FRANCE}

Introduction: Suicide prevention is a public health priority in France with approximately 12,000 deaths by suicide (prevalence 24 cases for 100,000 inhabitants) and 150,000 suicide attempts per year. Patients having made a suicide attempt commonly have recourse to emergency departments. The purpose of this work is to analyse the networks of care that are proposed to them by the staffs of the emergency departments.

Method: A questionnaire was administered that sought data concerning the patient, the suicidal gesture, the patient's evolution, and the proposed networks of care recommended. The emergency departments that participated in this prospective study had to ensure the inclusion of 50 consecutive patients admitted for suicide attempt.

Results: A total of 3,687 files from 77 emergency departments were abstracted. Characteristics of the suicidants were: female predominance (66\%), average age $36 \pm 14$ years, antecedent of suicide attempts (49\%), followed by a medical practitioner $(77 \%)$ or a psychiatrist $(43.5 \%)$, and voluntary drug ingestion (90\%). Non-hospitalised patients (18\%) left the emergency departments after receiving a psychiatric notice $(68 \%)$, against medical advice $(14 \%)$, or by escape $(9.5 \%)$. Twenty-nine percent of them left the emergency department without a directive, and $70 \%$ were referred to a psychiatrist (45\%) and/or to the family doctor (28\%) with an appointment in $27 \%$ of the cases. A prescription was delivered to $6.2 \%$ of non-hospitalised patients with, in half of cases, an antidepressant and/or an anxiolytic drugs. Hospitalizations ( $82 \%$ of patients) were provided in a short-term hospitalisation unit (69\%), in medical departments $(11 \%)$, in intensive care units $(9 \%)$, or in psychiatry (9\%); this varied according to the centers. The majority of patients left the hospital (67\%) after the initial hospitalization; except for psychiatry, the duration of hospitalization were 1 day and 2 days for respectively 69.5 and $84 \%$ of the patients with suicide attempts. During the hospitalization, $63 \%$ of patients met with a psychiatrist one time; services of a social worker were rarely requested (5\%)., No follow-up medical care has been proposed for $13.4 \%$ of the patients when they left hospital, and in $40 \%$ of cases, they were directed to a family doctor and/or a psychiatrist; the assistance of associations for suicidal patients only were requested as an exception. A prescription was given to $16.3 \%$ of patients with an antidepressant and/or an anxiolytic in $62 \%$ of cases. The main diagnoses obtained by psychiatrists have been "circumstantial crisis" $(46.6 \%)$, depression (37.6\%) with melancholy in $1.3 \%$ of depression cases, and psychosis (6.5\%).

Conclusion: The hospital management of suicide attempts is short and multidisciplinary. Nevertheless, psychological awakening beds have been created for the best networks of medical care.

Key words: attempts; care; causes; demography; disposition; emergency departments; gestures; hospitalizations; suicide; treatment

Prehosp Disast Med 2001;16(2):s71. 